\title{
Biological and clinical significance of MMP-2, MMP-9, TIMP-1 and TIMP-2 in non-small cell lung cancer
}

\author{
PILAR INIESTA ${ }^{1}$, ALBERTO MORÁN ${ }^{1}$, CARMEN DE JUAN ${ }^{1}$, ANA GÓMEZ ${ }^{2}$, FLORENTINO HERNANDO², \\ CRISTINA GARCÍA-ARANDA ${ }^{1}$, CRISTINA FRÍAS ${ }^{1}$, ANTONIO DÍAZ-LÓPEZ ${ }^{1}$, \\ FRANCISCO-JAVIER RODRÍGUEZ-JIMÉNEZ ${ }^{1}$, JOSE-LUIS BALIBREA ${ }^{2}$ and MANUEL BENITO ${ }^{1}$ \\ ${ }^{1}$ Departamento de Bioquímica y Biología Molecular, Facultad de Farmacia, Universidad Complutense; \\ ${ }^{2}$ Servicio de Cirugía, Hospital Clínico San Carlos, 28040 Madrid, Spain
}

Received August 30, 2006; Accepted October 2, 2006

\begin{abstract}
Our main aim consists of investigating the clinical usefulness of gelatinases and their tissue inhibitors in nonsmall cell lung cancer (NSCLC). Thus, we have analysed in 111 NSCLCs, levels and activity of MMP-2, MMP-9, TIMP-1 and TIMP-2, by Enzymoimmunoassay and Gelatine zymography, respectively. Our data revealed higher MMP-2 net activity in the NSCLC population analyzed in this study, this parameter showing a significant association with the TNM stage of tumours $(\mathrm{P}=0.002)$. Moreover, MMP-9 levels were significantly associated with poor clinical evolution of patients $(\mathrm{P}=0.02)$. Also, disease-free survival time was higher for patients whose tumours showed TIMP-1 increased levels $(\mathrm{P}=0.04)$. Of interest, Cox multivariate analysis revealed that TIMP-1 levels can be considered as an independent prognostic factor in NSCLC. Relative Risk (RR) to tumour relapse was more than two times lower for patients showing high TIMP-1 levels $(\mathrm{RR}=0.420, \mathrm{P}=0.041)$. Therefore, according to our results, we conclude that MMP-9 and TIMP-1 levels of synthesis could be useful for the selection of patients with potentially unfavourable clinical evolution in order to establish adjuvant therapy protocols. Among these parameters, TIMP-1 level evaluation emerges as the main factor to predict the clinical outcome of patients.
\end{abstract}

\section{Introduction}

Lung cancer has remained one of the most prevalent and deadly tumour types in both men and women. The high mortality rate for lung cancer results, at least in part, from the absence

Correspondence to: Professor Manuel Benito, Departamento de Bioquímica y Biología Molecular, Facultad de Farmacia, Universidad Complutense, 28040 Madrid, Spain

E-mail: benito@farm.ucm.es

Key words: non-small cell lung cancer, gelatinases, TIMP-1, TIMP-2, prognosis of standard clinical procedures for diagnosis of the disease at early and more treatable stages compared to other cancers. In spite of recent molecular advances in relation to factors that have been directly implicated in lung cancer progression, recurrence rates in the earliest pathological stages represent more than $40 \%$ of cases submitted to surgical resection (1). The recurrent propensity is linked to the cell capacity of degrading basement membranes and extracellular matrix. Matrix metalloproteinases (MMPs) are believed to be the main tissue-specific physiologically relevant mediators of matrix degradation (2). Gelatinases or type IV collagenases [72-kDa gelatinase (MMP-2) and 92-kDa gelatinase (MMP-9)] have been related to important roles in tumour invasion. Gelatinase A (MMP-2) is the most widely distributed of the MMPs and is expressed constitutively by most cells, including endothelial and epithelial cells. MMP-9 (gelatinase B) is produced by inflammatory cells as well as by stimulated connective tissue cells (3). MMP activity is precisely regulated at three levels: transcriptional expression; proteolytic activation, since these enzymes are secreted as a latent proenzyme form; and by the presence of tissue-specific inhibitors (TIMPs) (4). MMP-2 and MMP-9 have been identified in many human cancers both in neoplastic tissues and in the surrounding stromal and inflammatory cells (5).

Futhermore, there is evidence of a positive correlation between the progressive stages of cancer and the presence of gelatinases in different tumour types $(6,7)$. In vitro studies have demonstrated an association between the presence of these MMPs and their invasive potential (8), and prognostic implications for MMP-2 and MMP-9 have been referenced in some cancer types (9).

In lung cancer, the production of gelatinases and their natural tissue inhibitors TIMP-1 and TIMP-2 have been examined in cell lines derived from different histologic types, and gelatinolytic activity fits well with the clinical metastatic behaviour of small-cell lung cancer (10). Moreover, recent data suggests that advanced lung cancer does alter the normal circulatory pattern of MMP-9 and TIMP-1 (11). Also, expression profiles in stage I lung cancer revealed that MMP determination may provide a clue to the preselection of patients in clinical trials to investigate the benefits of MMP-inhibitory therapy (12). 
Thus, in the present study our aim consists of investigating the clinical usefulness of gelatinases and their tissue inhibitors in non-small cell lung cancer, considering the relevance of MMP-2 and MMP-9 in evaluating the potential metastatic behaviour of lung cancer. We have examined the production and activity of gelatinases, as well as their tissue inhibitors TIMP-1 and TIMP-2 in a wide non-small cell lung cancer (NSCLC) population that includes cancers classified into different stages related to the extension grade of this pathology.

\section{Materials and methods}

Patients and tissue processing. One hundred and eleven primary non-small cell lung carcinomas and their corresponding control tissue samples were obtained from patients who underwent surgery at San Carlos hospital in Madrid, Spain. Previously to surgery, none of these patients had received adjuvant treatment. Informed consent was obtained from patients prior to investigation. Of the 111 patients, 8 were female and 103 were male, with an average age of $63.56 \pm 9.55$ (range 40-83) years. After surgical resection, all tissue samples were snap-frozen in liquid nitrogen immediately after surgery and stored at $-80^{\circ} \mathrm{C}$ until processed. Cryostatsectioned, H\&E-stained samples from each tumour block were examined microscopically by two independent pathologists to confirm the presence of $>80 \%$ tumour cells. Paired normal tissues from the same patient, used as controls, were obtained at least $10 \mathrm{~cm}$ away from the margin of the tumour and also microscopically confirmed. Tumours were staged pathologically using the tumour node metastasis (TNM) system (13) and consisted of 67 TNM stage I, 8 TNM stage II, 25 TNM stage IIIA, 9 TNM stage IIIB, and 2 TNM stage IV tumours. According to the World Health Organization criteria, 61 tumours were squamous cell carcinomas (SCC), 46 were adenocarcinomas (AC), and 4 were large-cell undifferentiated carcinomas (LCUC). The histological classification of tumours was established according to previous criteria (14). Thus, 33 tumours were well-differentiated, 50 were moderately, and 28 were poorly differentiated. The median follow-up period for patients was 5 years (range, 1-79 months).

MMP-2, MMP-9, TIMP-1 and TIMP-2 levels by Enzymoimmunoassay. MMP-2 and MMP-9 protein total levels were determined from normal and tumour sample homogenates by Enzymoimmunoassay using kits obtained from Oncogene Research Products (Cambridge, MA). The total protein concentration of each sample was determined by the method of Bradford. Duplicate tissue sample homogenates were diluted when necessary (MMP-2, 1:20 for non-tumour and tumour tissues; MMP-9, 1:20 for non-tumour tissues and 1:60 for tumour tissues; TIMP-1 and TIMP-2, 1:10 for both types of tissues). Before performing the assays, possible protein degradation was evaluated by determining alkaline phosphatase activity.

ELISAs were performed according to the manufacturer's instructions. Briefly, the MMP-2, MMP-9, TIMP-1 and TIMP-2 enzyme immunoassays used two antibodies. An antibody, specific for the corresponding human protein, was immobilised onto the surface of microtiter wells. The samples to be assayed and biotinylated detector monoclonal antibodies
Table I. MMP-9, MMP-2, TIMP-1 and TIMP-2 levels in control and tumour tissues.

\begin{tabular}{lccr}
\hline Variable & $\begin{array}{c}\text { NT ng MMP or } \\
\text { TIMP/mg total } \\
\text { protein (mean } \pm \text { SE) }\end{array}$ & $\begin{array}{c}\text { T ng MMP or } \\
\text { TIMP/mg total } \\
\text { protein (mean } \pm \text { SE) }\end{array}$ & P value \\
\hline MMP-9 & $30.90 \pm 2.08$ & $63.12 \pm 4.78$ & $<0.001$ \\
MMP-2 & $8.14 \pm 0.54$ & $12.16 \pm 0.99$ & $<0.001$ \\
TIMP-1 & $2.63 \pm 0.17$ & $8.65 \pm 1.05$ & $<0.001$ \\
TIMP-2 & $13.63 \pm 2.98$ & $6.92 \pm 0.84$ & 0.029 \\
\hline
\end{tabular}

NT, non-tumour tissue; T, tumour tissue; SE, standard error.

were pipetted into the wells and allowed to incubate for $2 \mathrm{~h}$, during which time any MMP-2, -9 , TIMP-1 or -2 present in the sample bound to the capture and detecting antibodies. Unbound material was washed away, and horseradish peroxidase-conjugated streptavidin was added. The horseradish peroxidase catalysed the conversion of the chromogenic substrate tetramethylbenzidine from a colourless solution to a coloured solution, the intensity of which is proportional to the amount of the corresponding protein in the sample. The coloured reaction products were quantified using a microplate reader set at $450 \mathrm{~nm}$ (Benchmark; Bio-Rad). Quantitations were achieved by the construction of standard curves using known concentrations of MMP-2, MMP-9, TIMP-1 or TIMP-2.

Gelatine zymography. Gelatine zymography was performed on all samples as adapted from the method of Heussen and Dowdle (15). In brief, $8 \%$ sodium dodecyl sulfate (SDS)polyacrylamide electrophoresis gels copolymerized with $1 \mathrm{mg} /$ $\mathrm{ml}$ gelatine were used to detect both latent and activated forms of gelatinases. An aliquot of $50 \mu \mathrm{g}$ of total protein was separated by electrophoresis under non-denaturing conditions. Following electrophoresis, gels were washed twice in $2.0 \%$ Triton X-100 for $30 \mathrm{~min}$ at room temperature with shaking to remove SDS. Zymograms were subsequently developed by incubation overnight at $37^{\circ} \mathrm{C}$ in collagenase buffer $[0.2 \mathrm{M}$ $\mathrm{NaCl}, 5 \mathrm{mM} \mathrm{CaCl}_{2}, 1 \%$ (v/v) Triton X-100 and $0,02 \% \mathrm{NaN}_{3}$ in $50 \mathrm{mM}$ Tris-HCl, pH 7.4]. Zymograms were stained with $1 \mathrm{wt} / \mathrm{vol}$ Coomassie blue G-250 dissolved in 30\% methanol containing $10 \% \mathrm{v} / \mathrm{v}$ glacial acetic acid at room temperature for $60 \mathrm{~min}$. Gels were destained in the same solution but without the Coomassie blue stain. Recombinant human proteins were used as gelatinase controls.

Gelatinolytic activity was visualised as a clear band against a dark background of stained gelatine and quantitated by densitometry using a Bioimage Analyzer (GS-710; Bio-Rad) linked to Quantity One software (Bio-Rad). The enzyme activity levels are expressed as the fold-increase in the expression of the 92-, 82-, 72- and 62-kDa bands in tumour relative to that measured in the corresponding adjacent normal tissue.

Statistical analysis. All computations were carried out with SPSS, version 11.5. Differences among multiple groups, and 
Table II. MMP-9, MMP-2, TIMP-1 and TIMP-2 synthesis fold-increase in tumour versus non-tumour samples, in relationship to clinical pathological variables in non-small cell lung cancer.

\begin{tabular}{|c|c|c|c|c|c|c|c|c|c|}
\hline Variable & No. of cases & MMP-9 & $\mathrm{P}$ value & MMP-2 & $\mathrm{P}$ value & TIMP-1 & $\mathrm{P}$ value & TIMP-2 & $P$ value \\
\hline TNM stage & & & 0.933 & & 0.249 & & 0.888 & & 0.073 \\
\hline I & 67 & $4.76 \pm 1.92$ & & $2.49 \pm 0.34$ & & $4.01 \pm 0.46$ & & $0.97 \pm 0.16$ & \\
\hline II & 8 & $2.52 \pm 0.42$ & & $1.00 \pm 0.23$ & & $4.08 \pm 0.65$ & & $0.28 \pm 0.07$ & \\
\hline IIIA & 25 & $2.45 \pm 0.52$ & & $1.84 \pm 0.32$ & & $4.32 \pm 0.96$ & & $1.75 \pm 0.53$ & \\
\hline IIIB & 9 & $3.17 \pm 0.65$ & & $1.18 \pm 0.18$ & & $2.78 \pm 0.47$ & & $2.61 \pm 1.50$ & \\
\hline IV & 2 & $1.54 \pm 0.87$ & & $0.70 \pm 0.52$ & & $2.59 \pm 1.79$ & & $0.15 \pm 0.06$ & \\
\hline Histological type & & & 0.001 & & 0.654 & & 0.808 & & 0.616 \\
\hline Squamous cell carcinoma & 61 & $2.73 \pm 0.42$ & & $2.00 \pm 0.23$ & & $4.01 \pm 0.48$ & & $1.17 \pm 0.27$ & \\
\hline Adenocarcinoma & 46 & $2.69 \pm 0.65$ & & $1.81 \pm 0.27$ & & $4.16 \pm 0.76$ & & $1.34 \pm 0.36$ & \\
\hline $\begin{array}{l}\text { Large cell undifferentiated } \\
\text { carcinoma }\end{array}$ & 4 & $33.65 \pm 31.52$ & & $1.25 \pm 0.68$ & & $2.78 \pm 0.54$ & & $0.27 \pm 0.09$ & \\
\hline Differentiation & & & 0.383 & & 0.992 & & 0.120 & & 0.896 \\
\hline Well & 33 & $3.41 \pm 0.75$ & & $1.96 \pm 0.39$ & & $5.16 \pm 0.88$ & & $1.36 \pm 0.38$ & \\
\hline Moderately & 50 & $2.79 \pm 0.53$ & & $1.90 \pm 0.27$ & & $4.08 \pm 0.63$ & & $1.29 \pm 0.31$ & \\
\hline Poorly & 28 & $7.32 \pm 5.27$ & & $1.94 \pm 0.33$ & & $2.89 \pm 0.36$ & & $1.09 \pm 0.47$ & \\
\hline
\end{tabular}

potential associations with clinic pathological parameters were assessed using the chi-square test. A P value $<0.05$ was judged to be significant. Disease-free survival time (DFS) was estimated from date of surgery to date to tumour relapse. Thus, distributions of DFS were performed with the KaplanMeier method, and comparisons were made with log-rank statistics. Results were considered significant for $\mathrm{P}$ values $<0.05$. For survival analysis, only patients who had undergone potentially curative surgery (patients with TNM stage I-IIIA tumours) were considered. Also excluded were the 8 patients who died in the postoperative period. Thus, the number of patients included in the survival study was 92 .

Multivariate analysis was performed using the Cox proportional hazards model to identify which independent factors jointly had a significant influence on survival.

\section{Results}

From Enzymoimmunoassays, our data indicated that MMP-9, MMP-2 and TIMP-1 levels were significantly greater in primary non-small cell lung tumours than in the corresponding control samples. As can be observed in Table I, MMP-9 and MMP-2 synthesis in tumours versus non-tumour tissues showed respectively 2.04- and 1.49-fold increase $(\mathrm{P}<0.001$ in both cases). For TIMP-1 levels, our data revealed a 3.29fold increase in tumours versus control samples $(\mathrm{P}<0.001)$. Moreover, our results for the tissue inhibitor TIMP-2 indicated significantly lower levels in tumour samples (1.97-fold decrease, $\mathrm{P}=0.029$ ).

Statistical analyses of the synthesis levels in tumours compared to normal samples for MMP-9, MMP-2, TIMP-1 and TIMP-2 did not show significant associations with TNM stage or differentiation degree. However, histology of tumours was significantly associated with MMP-9 synthesis $(\mathrm{P}=0.001$,
Table II), LCUC displaying the highest levels for this metalloproteinase.

MMP activity in lung cancer was examined by gelatinolitic assays. Thus, zymographic analyses detected the latent proforms (Mr $72 \mathrm{kDa}$ and $92 \mathrm{kDa}$ ) and activated enzyme forms (Mr $62 \mathrm{kDa}$ and $82 \mathrm{kDa}$ ) of MMP-2 and MMP-9. From Gelatine zymography, a positive correlation was obtained both in the case of pro-MMP9 and MMP-9 activity ( $\mathrm{P}=0.01$, Pearson correlation), and in the case of pro-MMP-2 and MMP-2 activity ( $\mathrm{P}=0.01$, Pearson correlation). Likewise, correlation between MMP-9 and MMP-2 active forms and levels of synthesis for these MMPs were significant $(\mathrm{P}<0.001$ and $\mathrm{P}=0.009$, respectively).

Next, we considered the effect of TIMP-1 and TIMP-2 levels on the activity of MMP-9 and MMP-2 respectively. For this purpose, we defined the concept of net MMP activity by establishing the relationship between MMP active forms and TIMP levels in tumour tissues. Thus, the ratio MMP-9 active form/TIMP-1 level was $2.91 \pm 0.73$, compared with $4.60 \pm 0.86$ from the relation MMP-2 active form/TIMP-2 levels. Therefore, our data revealed higher net activity levels for MMP-2 in the NSCLC population studied. MMP net activity was related to clinic pathological variables of tumours without significant associations.

In addition, borderline correlations were detected between lymph node metastasis and several MMP-related parameters (Table III). Thus, higher levels of MMP-9, TIMP-1 or TIMP-2 synthesis were observed in cases showing positive lymph node metastasis $(\mathrm{P}=0.090 ; \mathrm{P}=0.086 ; \mathrm{P}=0.073$, respectively). Moreover, MMP-2 net activity was approximately 2-fold increased in cancers in which lymph node involvement was detected $(\mathrm{P}=0.09)$.

Finally, survival studies were performed using the KaplanMeier method. For these analyses we considered DFS time, 
Table III. Lymph node metastasis and MMPs or TIMPs in non-small cell lung cancer.

\begin{tabular}{lrrr}
\hline & \multicolumn{3}{c}{ Lymph node metastasis } \\
\cline { 2 - 4 } & \multicolumn{1}{c}{$+(\mathrm{n}=71)$} & $-(\mathrm{n}=40)$ & P value \\
\hline${\text { MMP-9 } \text { synthesis }^{\mathrm{a}}}$ & $74.11 \pm 9.11$ & $57.31 \pm 5.43$ & 0.090 \\
MMP-9 net activity $^{\mathrm{b}}$ & $3.52 \pm 1.65$ & $2.60 \pm 0.65$ & 0.550 \\
TIMP-1 synthesis $^{\mathrm{a}}$ & $11.18 \pm 2.71$ & $7.40 \pm 0.64$ & 0.086 \\
MMP-2 synthesis $^{\mathrm{a}}$ & $13.40 \pm 1.88$ & $11.40 \pm 1.16$ & 0.343 \\
MMP-2 net activity $^{\mathrm{b}}$ & $6.54 \pm 1.79$ & $3.48 \pm 0.87$ & 0.090 \\
TIMP-2 synthesis $^{\mathrm{a}}$ & $7.22 \pm 1.65$ & $6.68 \pm 0.94$ & 0.073 \\
\hline
\end{tabular}

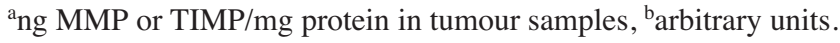

Table IV. Groups established to analyse clinical outcome of patients affected by NSCLC.

\begin{tabular}{|c|c|c|}
\hline Variable & Group 1 (G1) & Group 2 (G2) \\
\hline MMP-9 total levels ${ }^{a}$ & $<48.50$ & $\geq 48.50$ \\
\hline MMP-2 total levels ${ }^{\mathrm{a}}$ & $<9.39$ & $\geq 9.39$ \\
\hline TIMP-1 total levels ${ }^{\mathrm{a}}$ & $<6.22$ & $\geq 6.22$ \\
\hline TIMP-2 total levels ${ }^{\mathrm{a}}$ & $<3.87$ & $\geq 3.87$ \\
\hline $\begin{array}{l}\text { Fold-increase in MMP-9 } \\
\text { synthesis }^{\text {b }}\end{array}$ & $<2.18$ & $\geq 2.18$ \\
\hline $\begin{array}{l}\text { Fold-increase in MMP-2 } \\
\text { synthesis }^{\text {b }}\end{array}$ & $<1.38$ & $\geq 1.38$ \\
\hline $\begin{array}{l}\text { Fold-increase in TIMP-1 } \\
\text { synthesis }^{\text {b }}\end{array}$ & $<2.98$ & $\geq 2.98$ \\
\hline $\begin{array}{l}\text { Fold-increase in TIMP-2 } \\
\text { synthesis }^{\text {b }}\end{array}$ & $<0.56$ & $\geq 0.56$ \\
\hline net MMP-9 activity & $0(-)$ & $>0(+)$ \\
\hline net MMP-2 activity ${ }^{c}$ & $0(-)$ & $>0(+)$ \\
\hline
\end{tabular}

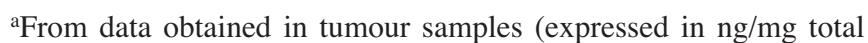
protein); bexpressed as values in tumour tissues as comparing to values in non-tumour samples; ${ }^{\text {net }}$ MMP activity = MMP active form in tumour tissues/TIMP levels (ng/mg total protein).

according to criteria that have been specified in the Materials and methods section. Thus, the impact on patient prognosis of MMP-9, MMP-2, TIMP-1 and TIMP-2 total levels in tumour samples, as well as fold-increases in synthesis in tumours versus control samples, or MMP-9 and MMP-2 net activity were investigated. For each one of the markers, we established two groups after comparing mean levels of variables across quartiles (Table IV). Thus, for each one of the variables, Group 1 (G1) included the cases of the quartiles 1 and 2, and Group 2 (G2) corresponded to quartiles 3 and 4.

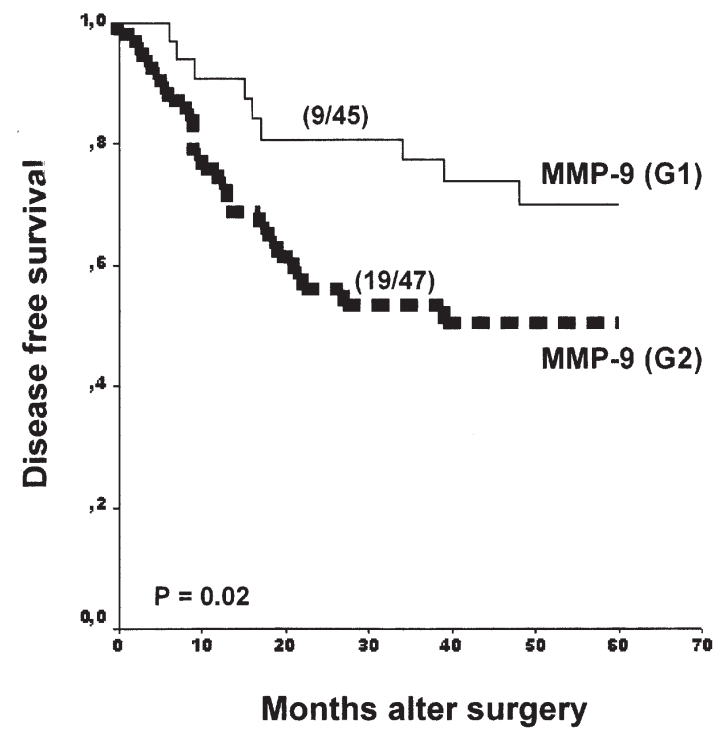

Figure 1. Kaplan-Meier survival curves for non-small cell lung cancer patients in function of MMP-9 tumour levels. G1, Group 1 including quartiles 1 and 2; G2, Group 2 including quartiles 3 and 4 .

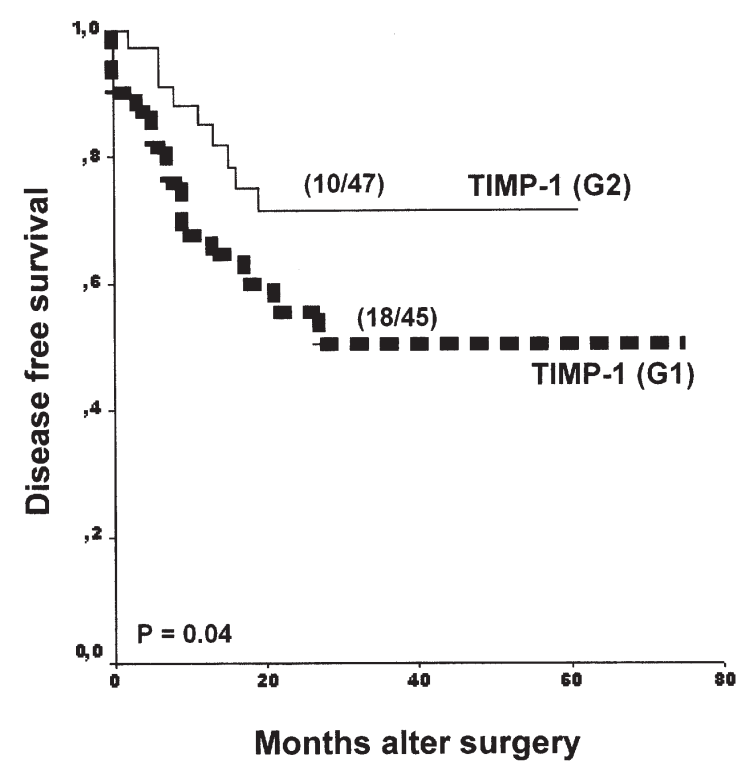

Figure 2. Survival curves showing clinical outcome in patients with nonsmall cell lung tumours in relation to TIMP-1 levels. G1, Group 1 including quartiles 1 and 2; G2, Group 2 including quartiles 3 and 4 .

In the context of variables considered as potential clinical tools in non-small cell lung cancer, our data revealed that MMP-9 tumour increased synthesis and TIMP-1 tumour decreased synthesis emerged as prognostic relevance parameters. As can be observed in Fig. 1, synthesis increased values in MMP-9 were significantly associated with poor clinical evolution of patients ( $\mathrm{P}=0.02$, by log-rank test). Also, disease-free survival time was higher for patients whose tumours showed TIMP-1 increased levels ( $\mathrm{P}=0.04$, Fig. 2). Of interest, as shown in Table $\mathrm{V}$, Cox multivariate analysis revealed that TIMP-1 synthesis fold-increase, in tumours versus non-tumour tissues, can be considered as an independent prognostic factor in NSCLC. In fact, TIMP-1 emerged as a 
Table V. Cox multivariate analysis for TNM stage and MMP-9 or TIMP-1 synthesis.

\begin{tabular}{lccc}
\hline Variable & RR & $\begin{array}{c}95 \% \text { confidence } \\
\text { interval }\end{array}$ & P value \\
\hline TNM stage & 1.985 & $1.276-3.089$ & $\mathbf{0 . 0 0 2}$ \\
$\begin{array}{l}\text { MMP-9 synthesis fold } \\
\text { increases in T vs NT }\end{array}$ & 2.069 & $0.938-4.563$ & 0.072 \\
$\begin{array}{l}\text { TIMP-1 synthesis fold } \\
\text { increase in T vs NT }\end{array}$ & 0.420 & $0.183-0.964$ & 0.041 \\
\hline \begin{tabular}{l} 
T, tumour samples; NT, non-tumour samples; RR, relative risk. \\
\hline
\end{tabular}
\end{tabular}

significant protective variable independent of tumour stage. Relative Risk (RR) was more than two times lower than in the case of tumours with lower TIMP-1 levels $(R R=0.420$, $\mathrm{P}=0.041$ ). Moreover, according to the Cox proportional hazard model, MMP-9 synthesis can be considered as a borderline independent prognostic biomarker. A high rate of relapse was detected in patients who had tumours in which MMP-9 synthesis was increased $(\mathrm{RR}=2.069, \mathrm{P}=0.072)$.

\section{Discussion}

Lung cancer is the commonest cause of cancer death in Europe and the United States. Despite advances in surgery, chemotherapy, and radiotherapy in the last two decades, the death rate has remained little changed. A minority of patients with NSCLC have tumours sufficiently localized to be considered treatable by surgical resection, and among those whose tumours are successfully resected, approximately $50 \%$ survive 5 years (16). According to reported data (17) clearly some NSCLCs have developed occult spread beyond the lung even when they appear to have been completely removed. Thus, if it would be possible to identify those tumours destined to relapse, adjuvant chemotherapy could be more successful in eradicating any residual tumour.

Matrix Metalloproteinases (MMPs), especially MMP-2 and MMP-9, are known to play a role in angiogenesis, tumour growth and metastasis $(18,19)$. Thus, it has been described that proMMP-2 and proMMP-9 expression is increased in malignant cancers compared to benign cancers (20-22). Recently, some studies regarding MMP levels in lung cancer have been performed $(17,23,24)$ and revealed several promising therapeutic approaches to inhibit MMPs. However, additional results are necessary to better elucidate the clinical usefulness of MMPs in lung cancer. In this context, in the present study, a wide non-small cell lung cancer population has been evaluated in order to investigate the clinical relevance of MMP-2 and MMP-9, and their tissue inhibitors in NSCLC. We have analyzed MMP-2 and MMP-9 levels and activity for proforms and active forms, as well as TIMP-1 and TIMP-2 levels.

Our data indicated a significant increased synthesis for MMP-2, MMP-9 and TIMP-1 in tumour samples versus control tissues. However, TIMP-2 levels were significantly decreased in cancers as compared to normal samples. As has been previously described in other tumour types (9) the higher proteinase levels in cancers imply that these tissues are capable of causing proteolytic degradation of the surrounding extracellular matrix and this may affect the tumours' potential for invasion and metastasis. Also, according to previous data (20), our results seem to indicate a clear contribution of TIMP-1 in NSCLC to the down-regulation of MMP-9. However, in the case of TIMP-2 it is necessary to consider the specific role described for this tissue inhibitor in relation to the proMMP-2 activation (25).

Regarding clinical pathological characteristics of tumours, we found an interesting association between MMP-9 levels and histology, large-cell undifferentiated carcinomas (LCUC) displaying the higher levels for MMP-9. The malignant potential of LCUC far exceeds other types of the non-small cell carcinoma group $(2,3)$. Thus, our results could indicate that the elevated metastatic potential associated to this histological group of lung cancer may be influenced by its increased levels of the metalloproteinase MMP-9.

It is known that the down-regulation of MMPs may occur at the levels of transcriptional regulation of the genes, activation of secreted proenzymes, and interaction with TIMPs (4). Thus, taking into account these regulation points, we firstly analyzed the correlation between MMP-2 and MMP-9 active forms and their levels of synthesis, our results indicating a positive correlation between these parameters. Next, in order to evaluate the impact of the presence of MMP tissue inhibitors, we determined net MMP activity, using the relationship between MMP activity and the corresponding TIMP, as had previously been studied (9). Our data revealed higher MMP-2 net activity in the NSCLC population analyzed in the present study, this parameter showing a significant association with TNM stage of tumours. Moreover, among the clinical variables considered, lymph node metastasis showed borderline association with MMP-2 net activity. Positive lymph node metastasis also correlated with the higher levels of MMP-9, TIMP-1 or TIMP-2. Therefore, after establishing the relationship with clinical variables, our data in NSCLC indicated a correlation between MMP-2 net activity and clinical parameters considered as tools for describing the extent of tumour spread, such as TNM stage or lymph node metastasis. Although MMP-2 and MMP-9 are very similar proteinases, their contribution to biological or pathological processes can be very different and it is unclear which of the two, MMP-2 or MMP-9, is most important in tumour progression and metastasis. We did not obtain significant results for MMP-9 net activity in relation to clinical variables of tumours, despite the higher levels of both MMP-9 and TIMP-1 being clearly associated with positive lymph node metastasis in a similar manner to TIMP-2. At this point, it is necessary to consider the role of TIMP-1 inhibiting MMP-9, and the dual function proposed for TIMP-2, this tissue inhibitor being associated with both MMP-2 activation and its activity inhibition (25).

Finally, to perform a more exact evaluation of the impact of MMP-2, MMP-9, TIMP-1, and TIMP-2 on prognosis of patients, we established survival analyses considering the disease-free survival time of patients. These studies indicated 
that, in the context of variables included in this study being potential clinical tools in NSCLC, low levels of TIMP-1 in tumour samples constitute the best parameter to evaluate prognosis, since TIMP-1 decreased levels showed a significant correlation with the worst outcome of patients. This parameter emerged as an independent prognostic factor in NSCLC after performing the Cox multivariate analysis. In fact, TIMP-1 increased levels may be considered as a protective variable independent of tumour stage in patients who develop nonsmall cell lung cancer and are submitted to curative surgery. Only a previous study in non-small cell lung carcinomas had reported data on the possible clinical usefulness of TIMP-1 (26). In this study, TIMP-1 expression was evaluated by immunochemical techniques, and results published showed an association of TIMP-1 overexpression with more advanced disease, which according to the authors may suggest a role in prognosis. In other tumour types, however, TIMP-1 increased synthesis has been proposed as an indicator of good clinical evolution of patients (9). Moreover, our Kaplan-Meier survival study also revealed a clinical usefulness for MMP-9 levels of synthesis. In this respect, our data indicated an association between higher MMP-9 levels and a poor outcome of patients, this parameter emerging as an independent prognosis factor in NSCLC. Regarding our results from the Cox multivariate analysis, the group of patients showing MMP-9 increased levels in tumour samples had a risk two-fold higher than those with lower MMP-9 levels. In previous studies, investigating levels of circulating matrix metalloproteinase-9 in non-small cell lung cancer patients, the clinical value of this proteinase as a marker of metastasis and in the follow-up of lung cancer patients was suggested (27). Also, Swinson et al (28) reported that MMP-9 expression was associated with poor prognosis in NSCLC. Nevertheless, none of these studies performed a complete MMP-9 investigation, analysing levels and activity for this metalloproteinase, as well as TIMP-1 evaluation.

From data obtained in the present study, we conclude that MMP-2 net activity, as well as MMP-9 and TIMP-1 levels of synthesis could be useful for the selection of patients with potentially unfavourable clinical evolution in order to establish adjuvant therapy protocols. Among these parameters, TIMP-1 level evaluation emerges as the main factor to predict the clinical outcome of patients.

\section{Acknowledgements}

This study was supported by grants from Red Respira C03/11, FIS PI020193, Fundación de Investigación Médica Mutua Madrileña, and Red temática de investigación cooperativa de centros de cáncer C03/010. We thank Dr A. Sánchez-Pernaute, and Professors A. Torres and E. Díaz-Rubio for their contribution to the project.

\section{References}

1. Hoffman PC, Mauer AM and Vokes EE: Lung cancer. Lancet 355: 479-485, 2000.

2. Murray GI: Matrix metalloproteinases: a multifunctional group of molecules. J Pathol 195: 135-137, 2001.

3. Foda HD and Zucker S: Matrix metalloproteinases in cancer invasion, metastasis and angiogenesis. Drug Discov Today 6: 478-482, 2001.
4. Murphy G, Willenbrock F, Crabbe T, O'Shea M, Ward R, Atkinson S, O'Connell J and Docherty A: Regulation of matrix metalloproteinases activity. Ann NY Acad Sci 732: 31-41, 1994.

5. Crawford HC and Matrisian LM: Tumor and stromal expression of matrix metalloproteinases and their role in tumor progression. Invasion Metastasis 95: 234-245, 1994.

6. Davies B, Waxman J, Wasan H, Abel P, Williams G, Krausz T, Neal D, Thomas D, Hanby A and Balkwill F: Levels of matrix metalloproteinases in bladder cancer correlate with tumor grade and invasion. Cancer Res 53: 5365-5369, 1993.

7. Stearns ME and Wang M: Type IV (Mr 72,000) expression in human prostate: Benign and malignant tissue. Cancer Res 53: 878-883, 1993.

8. Kawahara E, Okada Y, Nakanashi I, Iwata K, Kojima S, Kumagai $S$ and Yamamoto $E$ : The expression of invasive behavior of differentiated squamous carcinoma cell line evaluated by an in vitro invasion model: Jpn J Cancer Res 84: 409-418, 1993.

9. Morán A, Iniesta P, García-Aranda C, de Juan C, Díaz-López A, Sánchez-Pernaute A, Torres AJ, Díaz-Rubio E, Balibrea JL and Benito M: Clinical relevance of MMP-9, MMP-2, TIMP-1 and TIMP-2 in colorectal cancer. Oncol Rep 13: 115-120, 2005.

10. González-Ávila G, Iturria C, Vadillo F, Terán L, Selman M and Pérez-Tamayo R: 72-kD (MMP-2) and 92-kD (MMP-9) Type IV Collagenase production and activity in different histologic types of lung cancer cells. Pathobiology 66: 5-16, 1998.

11. Jumper C, Cobos E and Lox C: Determination of the serum matrix metalloproteinase-9 (MMP-9) and tissue inhibitor of matrix metalloproteinase-1 (TIMP-1) in patients with either advanced small-cell lung cancer or non-small-cell lung cancer prior to treatment. Respir Med 98: 173-177, 2004.

12. Cho NH, Hong KP, Hong SH, Kang S, Chung KY and Cho SH: MMP expression profiling in recurred stage IB lung cancer. Oncogene 23: 845-851, 2004.

13. Mountain CF: A new international staging system for lung cancer. Chest 89 (suppl): 225-233, 1986.

14. Sobin L: The World Health Organization's histological classification of lung tumors: a comparison of the first and second editions. Cancer Detect Prev 5: 391-406, 1982.

15. Heussen C and Dowdle E: Electrophoretic analysis of plasminogen activators in polyacrylamide gels containing sodium dodecyl sulphate and copolymerised substrates. Anal Biochem 102: 196-202, 1980.

16. Strauss GM, Kwiatkowski DJ, Harpole DH, Lynch TJ, Skarin AT and Sugarbaker DJ: Molecular and pathologic markers in stage I non-small cell carcinoma of the lung. J Clin Oncol 13: 1265-1279, 1995.

17. Pinto CA, de Oliveira Carvalho PE, Antonângelo L, Garippo A, Pereira da Silva AG, Soares F, Younes R, Beyruti R, Takagaki T, Saldiva P, Vollmer RT and Capelozzi VL: Morphometric evaluation of tumor matrix metalloproteinase 9 predicts survival after surgical resection of adenocarcinoma of the lung. Clin Cancer Res 9: 3098-3104, 2003.

18. Coussens LM, Fingleton B and Matrisian LM: Matrix metalloproteinase inhibitors and cancer: trials and tribulations. Science 295: 2387-2392, 2002.

19. Egeblad M and Werb Z: New functions for the matrix metalloproteinases in cancer progression. Nat Rev Cancer 2: 161-174, 2002 .

20. Schmalfeldt B, Prechtel D, Harting K, Spathe K, Rutke S, Konik E, Fridman R, Berger U, Schmitt M, Kuhn W and Lengyel E: Increased expression of matrix metalloproteinases MMP-2, MMP-9, and the urokinase-type plasminogen activator is associated with progression from benign to advanced ovarian cancer. Clin Cancer Res 7: 2396-2404, 2001.

21. Hanemaaijer R, Verheijen JH, Maguirre TM, Visser H, Toet K, McDermott E, O'Higgins N and Duffy MJ: Increased gelatinase$A$ and gelatinase-B activities in malignant vs. benign breast tumors. Int J Cancer 86: 204-207, 2000.

22. Sheen-Chen SM, Chen HS, Eng HL, Sheen CC and Chen WJ: Serum levels of matrix metalloproteinase 2 in patients with breast cancer. Cancer Lett 173: 79-82, 2001.

23. Ohbayashi H: Matrix metalloproteinases in lung diseases. Curr Protein Pept Sci 3: 409-421, 2002.

24. Kim SJ, Rabbani ZN, Dewhirst MW, Vujaskovic Z, Vollmer RT, Schreiber EG, Oosterwijk E and Kelley MJ: Expression of HIF-1, CA IX, VEGF, and MMP-9 in surgically resected non-small cell lung cancer. Lung Cancer 49: 325-335, 2005. 
25. Caterina JJ, Yamada S, Caterina NC, Longenecker G, Holmback K, Shi J, Yermovsky AE, Engler JA and Birkedal-Hansen H: Inactivating mutation of the mouse tissue inhibitor of metalloproteinases-2 (TIMP-2) gene alters proMMP-2 activation. J Biol Chem 275: 26416-26422, 2000.

26. Thomas P, Khokha R, Shepherd FA, Feld R and Tsao MS: Differential expression of matrix metalloproteinases and their inhibitors in non-small cell lung cancer. J Pathol 190: 150-156, 2000.
27. Kaya A, Gulbay BE, Gurkan OU, Celik G, Savas H and Savas I: Elevated levels of circulating matrix metalloproteinase-9 in non-small cell lung cancer patients. Tuberk Toraks 51: 380-384, 2003.

28. Swinson DE, Cox G and O'Byrne KJ: Coexpression of epidermal growth factor receptor with related factors is associated with a poor prognosis in non-small-cell lung cancer. Br J Cancer 91: 1301-1307, 2004. 